\title{
Effectiveness of virtual reality immersion on procedure-related pain and anxiety in outpatient pain clinic: an exploratory randomized controlled trial
}

\author{
Young Joo ${ }^{1, *}$, Eun-Kyung Kim ${ }^{2, *}$, Hyun-Gul Song ${ }^{2, *}$, Haesun Jung ${ }^{3}$, HanssI Park ${ }^{2}$, and Jee Youn Moon ${ }^{2,4}$ \\ 'Department of Anesthesiology and Pain Medicine, CHA Ilsan Medical Center, CHA University School of Medicine, Goyang, Korea \\ ${ }^{2}$ Department of Anesthesiology and Pain Medicine, Seoul National University Hospital, Seoul, Korea \\ ${ }^{3}$ Department of Anesthesiology and Pain Medicine, Chung-Ang University Hospital, Seoul, Korea \\ ${ }^{4}$ Department of Anesthesiology and Pain Medicine, Seoul National University College of Medicine, Seoul, Korea
}

Received March 22, 2021

Revised April 16, 2021

Accepted April 19, 2021

Handling Editor: Rohit Aiyer

\section{Correspondence}

Jee Youn Moon

Department of Anesthesiology and Pain Medicine, Seoul National University Hospital, Seoul National University College of Medicine, 101 Daehak-ro, Jongno-gu, Seoul 03080, Korea

Tel: +82-2-2072-2467

Fax: +82-2-747-5639

E-mail: jymoon0901@gmail.com

*These authors contributed equally to this work as co-first authors.

Previous presentation at conferences This article was presented at the 66th Korean Pain Society Scientific Meeting \& Training Course on May 26, 2018, in Changwon Exhibition Convention Center, Changwon, Korea.
Background: The study investigated virtual reality (VR) immersion in alleviating procedure-related pain in patients with chronic pain undergoing fluoroscopy-guided minimally-invasive intervention in a prone position at an outpatient clinic.

Methods: In this prospective randomized controlled study, 38 patients undergoing lumbar sympathetic ganglion block were randomized into either the VR or the control group. In the VR group, procedure-related pain was controlled via infiltration of local anesthetics while watching a 30-minute VR hypnotic program. In the control group, the skin infiltration alone was used, with the VR device switched off. The primary endpoint was an 11-point score on the numerical rating scale, indicating procedure-related pain. Patients' satisfaction with pain control, anxiety levels, the need for additional local anesthetics during the procedure, hemodynamic stability, and any adverse events were assessed.

Results: Procedure-related pain was significantly lower in the VR group (3.7 \pm 1.4$)$ than in the control group $(5.5 \pm 1.7 ; P=0.002)$. Post-procedural anxiety was lower in the VR group than in the control group $(P=0.025)$, with a significant reduction from pre-procedural anxiety $(P<0.001)$. Although patients' satisfaction did not differ significantly $(P=0.158)$ between the groups, a higher number of patients required additional local anesthetics in the control group $(n=13)$ than in the VR group ( $n=4 ; P=0.001$ ). No severe adverse events occurred in either group during the study.

Conclusions: VR immersion can be safely used as a novel adjunct to reduce procedural pain and anxiety during fluoroscopic pain intervention.

Key Words: Ambulatory Care Facilities; Anxiety; Autonomic Nerve Block; Chronic Pain; Ganglia, Sympathetic; Hypnotics and Sedatives; Minimally Invasive Surgical Procedures; Pain Perception; Pain, Procedural; Virtual Reality; Virtual Reality Exposure Therapy. (c) This is an open-access article distributed under the terms of the Creative Commons Attribution Non-Commercial License (http://creativecommons.org/licenses/by-nc/4.0/), which permits unrestricted non-commercial use, distribution, and reproduction in any medium, provided the original work is properly cited.

(c) The Korean Pain Society, 2021
Author contributions: Young Joo: Manuscript preparation, Statistical analysis, Manuscript revision; Eun-Kyung Kim: Study design, Data collection, Manuscript revision; Hyun-Gul Song: Data collection, Statistical analysis, Manuscript revision; Haesun Jung: Data collection, Manuscript revision; HanssI Park: Data collection; Jee Youn Moon: Study design, Statistical analysis, Manuscript preparation, Manuscript revision. 


\section{INTRODUCTION}

Pain has a negative effect on the physical and psychosocial dimensions of quality of life $[1,2]$. The history of medicine suggests novel strategies to alleviate pain in numerous diseases. Although pharmacological treatment is pivotal in pain management [3], non-surgical and minimally-invasive procedures play a significant role, especially in pain practice [4]. They can be rapidly conducted in an outpatient setting, lasting only a few minutes in a patient who is awake. However, in the context of medical care, it sounds paradoxical that procedural pain involving minimally-invasive procedures is intolerable beside the original pain [5]. During the procedure, insufficient pain control may result in adverse consequences for patients, such as aborted procedures, avoidance of future therapeutic intervention, poor recovery, and psychological trauma. Skin infiltration with local anesthetics (LA) could mitigate procedure-related pain; however, it is often insufficient for patients undergoing painful procedures, leading to general or unknown anxiety during the intervention. Although intravenous (IV) sedation could be used for some painful procedures, it is not always adequate in an outpatient pain setting due to respiratory depression or hemodynamic instability $[6,7]$.

Virtual reality (VR) is a non-invasive simulation that allows users to interact with a computer-generated artificial environment [8]. Unlike augmented reality, it is a fully digital experience that can either stimulate or alter the real world [8]. In the medical field, VR has been used to distract patients from pain during uncomfortable medical procedures, such as in patients undergoing burn and wound care [9-11]. It has also been a useful option to reduce pain and anxiety during orthopedic surgeries, pediatric vascular access, and dental procedures [12-15]. VR immersion also provided better results than IV sedation during endoscopic urologic surgery under spinal anesthesia [16]. Combined with previous findings, VR immersion may reduce procedure-related pain in patients undergoing minimallyinvasive spinal interventions. However, the role of VR immersion in an outpatient pain practice setting is seldom reported.

This study hypothesized that VR might improve procedure-related experiences as an effective adjunct to conventional skin infiltration. To this end, we investigated the role of VR immersion in alleviating procedural pain intensity and mitigation of unpleasant experiences in awake patients with chronic pain undergoing fluoroscopy-guided minimally-invasive pain interventions in a prone position.

\section{MATERIALS AND METHODS}

\section{Study design}

This prospective, exploratory randomized controlled trial was approved by the Institutional Review Board of Seoul National University Hospital (No. 1802-028-920) and registered in ClnicalTrials.gov (NCT03599479 released on 24 June 2018). This study complied with the Declaration of Helsinki and was conducted at a single pain management center between December 2018 and August 2019. Written informed consent was obtained from all participants before initiating the study.

Inclusion criteria were: (1) patients aged between 20 and 85 years; and (2) patients with at least a 3-month duration of chronic pain who were scheduled to undergo fluoroscopy-guided lumbar sympathetic ganglion block (LSGB) in an outpatient setting. In this study, LSGB was selected among various minimally-invasive pain interventions, a relatively painful procedure with moderate procedural pain [5]. Institutional protocol requires that patients stay in the outpatient pain operation room (OR) for at least 20 minutes to confirm a temperature increase after administering LA, which is adequate to experience the VR immersion.

Exclusion criteria were: (1) patients with visual or hearing impairment; (2) patients with previous psychotic, uncontrolled anxiety, or major affective disorders; (3) patients with cognitive defects or intellectual impairment; (4) patients with a disability that could affect adverse effect assessment or interfere with study completion when enrolled; (5) patients with a recent history of LSGB within 1 year before the randomization; (6) patients contraindicated for invasive procedures (coagulopathy, skin infection on the injection site, and allergies to LA); or (7) any patients who were considered inappropriate to register in this clinical trial.

\section{Randomization}

Randomization was conducted in an OR before starting the procedure. In the pre-operative holding room, all participants were educated in methods to handle the device with a brief experience of VR immersion for 2 minutes in a sitting position using a Samsung Gear head-mounted display compatible with the Android platform operating on a Galaxy 7.0 device (Samsung, Seoul, Korea). After entering the OR, patients were placed in a prone position, with their neck slightly flexed, and were assisted with putting on the VR head-mounted display and headphones. A soft gel pillow and a large foam pillow supported the patient's forehead and chest to prevent any pressure or discomfort from 

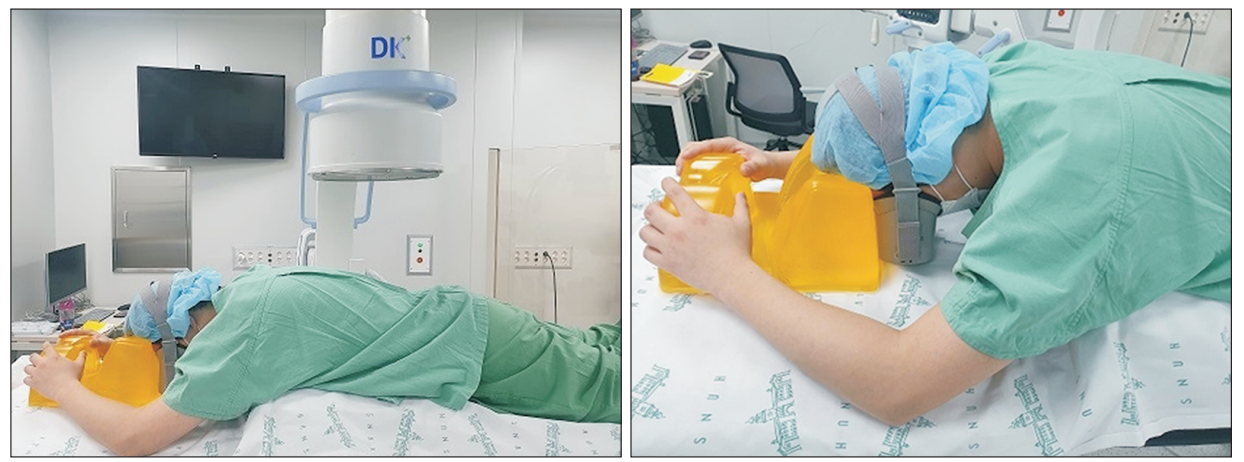

Fig. 1. Photographs of a person wearing the virtual reality device in a prone position to undergo the lumbar sympathetic ganglion block.

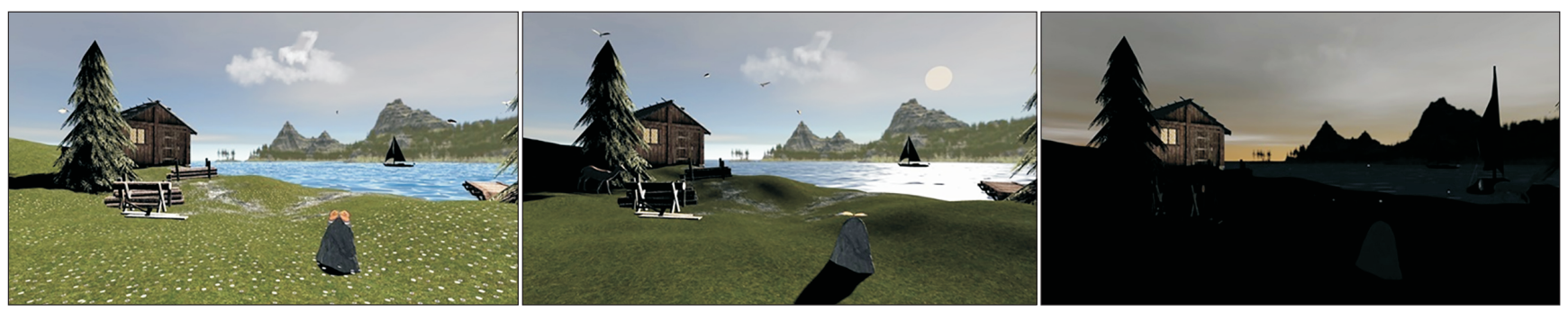

Fig. 2. Images of NUVO program by Oncomfort SA, Wavre, Belgium.

wearing the device (Fig. 1). The VR immersion began with proper fitting of VR device after repositioning the patients on the procedure bed. Next, the patients were randomly assigned to a VR group or a control group (1:1), based on a group allocation number within an opaque envelope opened by a pain fellow. The number in the envelope was matched to the group in a randomization table generated by an internet-based computer program (www.randomization.com), managed by a radio technician independent of the study. The patient was assisted with switching on the VR program in the test group or switching it off in the control group. A pain fellow was available to support patients throughout the patient's stay in the OR.

Patients in the VR group experienced a 30 -minute VR immersion (NUVO program by Oncomfort SA, Wavre, Belgium). The three-dimensional VR software consists of a seashore view with Korean language narrations designed to induce relaxation (Fig. 2). The program was developed initially as VR hypnosis to manage anxiety and pain during anxiety-provoking moments of treatment with portable immersive $360^{\circ}$ audio and video. During the VR immersion, the patient could feel relaxed, virtually sitting in front of the seashore. The patient could then move around and look at the scene from other views, enhancing physical and emotional comfort while listening to narrations designed to induce relaxation. The first author (EKK) corresponded with the program developers to translate the original English version into the Korean language. The VR experience started right after the group allocation, in order to create effective immersion in the hypnotic program.
Patients in the VR group were free to request discontinuation of the VR immersion at any time. In the control group, patients were asked to wear the VR head-mounted display and headphones, but the program was switched off while undergoing the procedure. During the procedure, the performing physician was blinded to which patients were in which group. The VR device was finally removed when the patient moved to the recovery room. Both groups could request removing their device at any time if they felt discomfort wearing the VR head-mount display and headphones.

\section{Procedures}

After entering the OR, patients were held in a prone position with a pillow under the lower abdomen, and draped in a sterile fashion. Other cushions were used to support the forehead and chest in patients wearing the VR headmounted display in a prone position. All patients were administered an IV infusion of lactated Ringer's solution and monitored via pulse oximetry, electrocardiogram (EKG), and blood pressure measurements throughout the procedure. In addition, temperature probes were tightly attached to both soles using transparent patches (Tega$\operatorname{derm}^{\mathrm{TM}}$, 3M Health Care, St. Paul, MN) before covering the patient's body to confirm the temperature increase in the ipsilateral lower extremity after the LSGB. After sterilizing the skin around the puncture sites, the body was covered by a sterile surgical drape. The procedure was performed under fluoroscopic guidance (OEC 9800 series; GE OEC Medical Systems, Salt Lake City, UT) by a single pain phy- 
sician (JYM) who had at least 10 years of experience to minimize inter-physician variation during the intervention. The pain physician was blinded to the patients' group allocation during the procedure and throughout the study. Skin infiltration with LA (2-3 mL of $1 \%$ lidocaine) was performed in both groups. At least 3 minutes after skin infiltration, a 21-gauge $15 \mathrm{~cm}$ Chiba needle (Cook Inc., Bloomington, IN) was advanced at the L3 vertebral level under fluoroscopy-guided oblique projection. If a patient complained of moderate-to-severe procedure-related pain while advancing the needle, additional LA was injected via the Chiba needle. The total amount of LA was less than $5 \mathrm{~mL}$, including skin infiltration, for procedural analgesia. When the needle reached the proper target site (anterolateral border of the L3 vertebral body), 1-2 $\mathrm{mL}$ of contrast agent was injected to confirm adequate spread around the target, followed by injection of $8 \mathrm{~mL}$ of $0.25 \%$ levobupivacaine. After removing the needle, temperature changes in the ipsilateral and contralateral soles were recorded for 20 minutes in the OR. The patients were then transferred to the recovery room.

\section{Data collection}

The primary outcome was the patient-reported procedural pain, which was measured using the 11-point numerical rating scale (NRS) on a scale of 0 (no pain) to 10 (the most severe pain imaginable). A research nurse recorded the pain scores immediately after the patients arrived in the recovery room.

The secondary outcomes included patient-reported anxiety before and after the procedure and patient-reported satisfaction with procedural pain control. The patientreported anxiety involving the procedure was assessed using the 5-point Likert scale (1, not at all anxious; 2 , a little anxious; 3 , moderately anxious; 4 , very anxious; and 5, extremely anxious) [17]. It was based on two questions: 1) "How anxious do you feel about your upcoming procedure right now?", which was asked before entering the OR, and 2) "Please imagine that you are supposed to undergo the same procedure right now. How anxious would you feel about the procedure at this moment?", which was asked in the recovery room 30 minutes after the procedure. At the same time, patient-reported satisfaction with control of procedure-related pain was also evaluated using a 5-point Likert scale (1, dissatisfied; 2 , less satisfied; 3 , satisfied; 4, very satisfied; and 5 , completely satisfied).

In the OR, the number of additive LA requirements (except for skin block) and any interruption of communication during the VR application were recorded. The total procedural time (min) was recorded, starting with the acquisition of the first radiographic image to the removal of the Chiba needle. The total VR time (min) was recorded, specifically starting from putting on the VR device in the OR to its removal. In the recovery room, subjective perception of stay ( $\mathrm{min}$ ) was evaluated by asking: "How much time were you supposed to stay in the OR?" Also, the patients in the VR group were asked about their subjective feedback on VR use and whether they would prefer VR immersion in their next pain procedures.

Safety evaluation was conducted via regular monitoring of any adverse events throughout the procedure. Pre- and post-procedural hemodynamic variables were compared using an average of 3 repeated measurements before the skin infiltration and before wearing off the VR device. Additionally, the intraoperative hemodynamic parameters were measured at regular intervals in the presence of bradycardia (heart rate $<40$ beats/min), hypotension (decrease in mean arterial pressure $<55 \mathrm{mmHg}$ or up to a $30 \%$ decrement from baseline), oxygen desaturation (SpO2 $<90 \%$ ), or provocation of arrhythmia on the EKG. Adverse effects due to VR immersion, such as dizziness, seizure, headache, or muscle twitching, were monitored until discharge.

Baseline demographic and clinical data included age, sex, body mass index, comorbidities (hypertension, diabetes mellitus, asthma, cardiovascular diseases, and cerebrovascular disease), comorbid neuropsychiatric disorders (depression and anxiety), the presence of motion sickness, educational level ( $<$ high school, high school, $>$ high school), duration of computer use ( $<1$ year, $1-5$ years, $>5$ years), diagnosis of pain, pain duration (month), baseline pain intensity (average level and the worst pain during the last week) based on an 11-point NRS pain score, and the use of strong opioids (morphine, oxycodone, hydromorphone, or fentanyl). Previous VR experience was evaluated dichotomously (yes or no). The patients' Hamilton Anxiety Rating (HAM-A) score was measured before the procedure. The HAM-A score is based on 14 individually rated items with the total score ranging from 0 to 54: a score of 14 or less indicates mild anxiety, a score ranging from 15 to 23 represents mild to moderate anxiety, and a score of 24 to 30 suggests moderate to severe anxiety [18].

\section{Statistical analysis and sample size justification}

In the absence of a preliminary study investigating the relevance of VR immersion to procedure-related pain scores in pain practice, we adopted a conventional strategy based on the differences in procedural pain between the two groups, suggested by Dworkin et al. [19], which was assumed to represent a significant decrease in the 11-point NRS pain score of 2.0 under clinical settings. It was comparable to a previous VR study of pain control during den- 
tal procedures, which reported that the procedural pain was 1.8/10.0 for those using VR and 4.0/10.0 for the control group [20]. We determined that 17 patients per group were necessary, assuming a standard deviation (SD) of 2.1 based on a previous study involving LSGB [5], with a type 1 error of 0.05 and a type 2 error of 0.2 . Allowing for $10 \%$ attrition, a total of 38 randomized patients were required for this study (G*Power 3.1.9.2).

All statistical analyses were performed using SPSS version 22.0 (IBM Co., Armonk, NY). The intention-to-treat approach was used for data analysis. To analyze continuous variables such as the primary endpoint (procedurerelated NRS pain scores), the normality distribution was determined with the Kolmogorov-Smirnov test and an independent $t$-test was used to compare the normally distributed variables. The Mann-Whitney $U$-test was used to compare continuous variables without normal distribution. A chi-square test or Fisher's exact test was used to analyze all categorical data. Lastly, we calculated the Spearman correlation coefficients $(\rho)$ to measure the correlation between the procedure-related NRS pain scores for the baseline and procedural variables. Data were presented as the mean \pm SD or the number (\%). All $P$ values are two-sided, and $P$ values less than 0.05 were considered to indicate statistical significance.

\section{RESULTS}

\section{Patient demographics and clinical characteristics}

A total of 45 patients were screened (Fig. 3). Two patients refused to participate and 5 patients had undergone LSGB or neurodestructive procedures within one year. The re- maining 38 patients were randomized to the VR group $(n=19)$ and control group $(n=19)$. All of the participants complied with the study protocol and were included in the analysis without missing data. The patient demographics and clinical characteristics are shown in Table 1. The groups showed no statistically significant differences in any demographic variables or comorbidities, or in the level of education or duration of computer use. And the patients' pain, etiology, pain onset, the average and most severe NRS pain scores at baseline, and their current use of strong opioids were comparable. Patients' baseline anxiety levels based on the HAM-A scores did not differ between the groups, suggesting moderate anxiety levels. Five patients had experienced a VR application beforehand $(\mathrm{n}=3$ in the VR group and $n=2$ in the control group); 1 in the VR group was familiar with VR technology.

\section{Procedural variables}

The primary endpoint of procedure-related NRS pain scores in the VR group were lower $(3.7 \pm 1.4)$ than in the control group $(5.5 \pm 1.7)$, which was statistically significant $(P=0.002)$ (Table 2). The 5-point patient-reported anxiety score involving the upcoming procedure did not differ between the groups before LSGB, suggesting moderate anxiety $(P=0.288)$; however, after the procedure, it was lower in the VR group $(2.5 \pm 0.8)$ than in the control group $(3.2 \pm 0.7)$ with statistical significance $(P=0.010)$. Patients in both groups reported "satisfied", or more, with pain control during the procedure, without a difference between the groups $(P=0.158)$. Seventeen patients $(44.7 \%)$ requested additional analgesia during the LSGB, which was more prevalent in the control group ( $\mathrm{n}=13,68.4 \%$ vs. $\mathrm{n}=4,21.1 \%$ in the VR group; $P=0.008$ ). The total duration

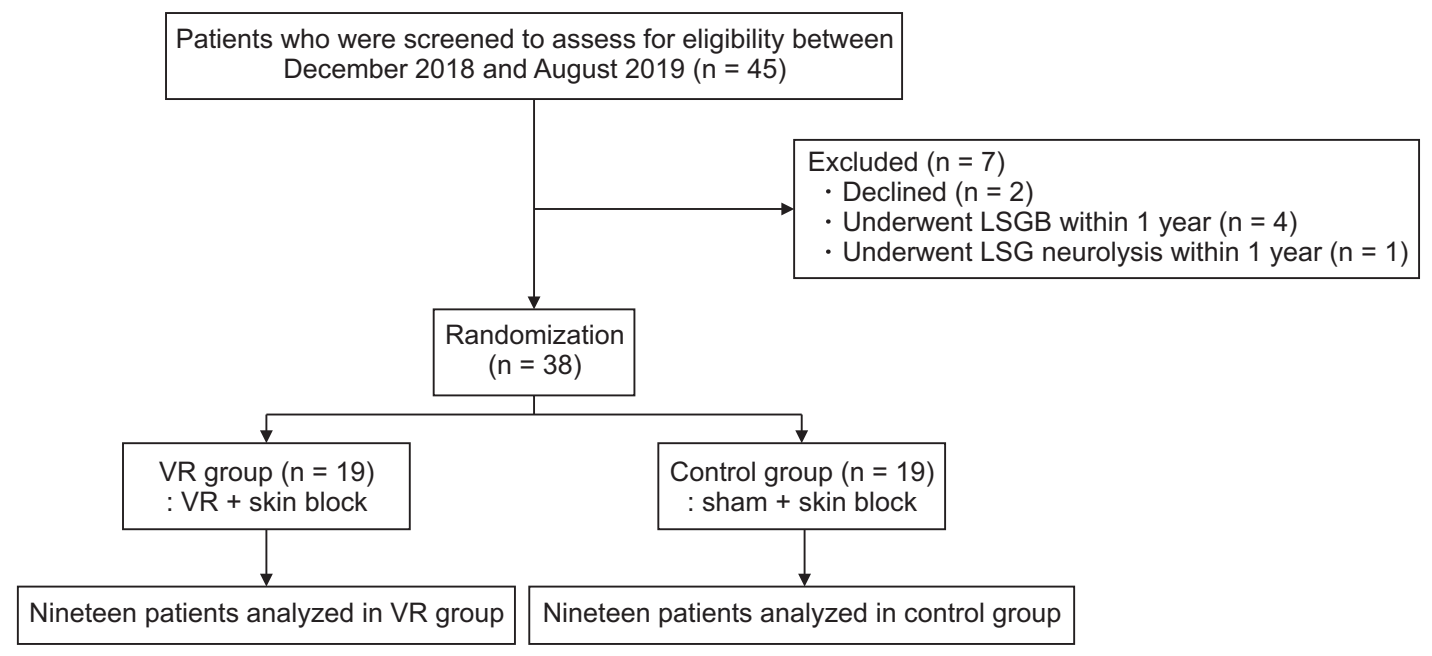

Fig. 3. Consolidated Standards of Reporting Trials (CONSORT) flow diagram. LSGB: lumbar sympathetic ganglion block, VR: virtual reality. 
Table 1. Patient demographics and clinical characteristics

\begin{tabular}{|c|c|c|c|}
\hline Variable & VR group $(n=19)$ & Control group $(n=19)$ & Total $(n=38)$ \\
\hline Age (yr) & $60.1 \pm 13.4$ & $65.2 \pm 12.9$ & $62.6 \pm 13.3$ \\
\hline Body mass index $\left(\mathrm{kg} / \mathrm{m}^{2}\right)$ & $24.2 \pm 4.1$ & $23.2 \pm 2.9$ & $23.7 \pm 3.5$ \\
\hline \multicolumn{4}{|l|}{ Sex } \\
\hline Male & $8(42.1)$ & $8(42.1)$ & $16(42.1)$ \\
\hline Female & $11(57.9)$ & $11(57.9)$ & $22(57.9)$ \\
\hline \multicolumn{4}{|l|}{ Education level } \\
\hline$<$ High school & $8(42.1)$ & $5(26.3)$ & $13(34.2)$ \\
\hline High school & $6(31.6)$ & $11(57.9)$ & $17(44.7)$ \\
\hline > High school & $5(26.3)$ & $3(15.8)$ & $8(21.1)$ \\
\hline \multicolumn{4}{|l|}{ Duration of computer utilization (yr) } \\
\hline$<1$ & $10(52.6)$ & $9(47.4)$ & $19(50.0)$ \\
\hline $1-5$ & $2(10.5)$ & $3(15.8)$ & $5(13.2)$ \\
\hline$>5$ & $7(36.8)$ & $7(36.8)$ & $14(36.8)$ \\
\hline Previous VR experience & $4(21.1)$ & $2(10.5)$ & $6(15.8)$ \\
\hline Motion sickness & $7(36.8)$ & $9(47.4)$ & $16(42.1)$ \\
\hline \multicolumn{4}{|l|}{ Comorbidities } \\
\hline Hypertension & $4(21.1)$ & $7(36.8)$ & $11(28.9)$ \\
\hline Diabetes mellitus & $4(21.1)$ & $4(21.1)$ & $8(21.1)$ \\
\hline Asthma & $1(5.3)$ & $1(5.3)$ & $2(5.3)$ \\
\hline Cardiovascular diseases & $3(15.8)$ & $1(5.3)$ & $4(10.5)$ \\
\hline Cerebrovascular diseases & $3(15.8)$ & $0(0.0)$ & $3(7.9)$ \\
\hline \multicolumn{4}{|l|}{ Neuropsychiatric disorders } \\
\hline Depression & $6(31.6)$ & $4(21.1)$ & $10(26.3)$ \\
\hline Anxiety & $3(15.8)$ & $5(26.3)$ & $8(21.1)$ \\
\hline \multicolumn{4}{|l|}{ Diagnosis } \\
\hline Complex regional pain syndrome & $6(31.6)$ & $7(36.8)$ & $13(34.2)$ \\
\hline Failed back surgery syndrome & $2(10.5)$ & $3(15.8)$ & $5(13.2)$ \\
\hline Postherpetic neuralgia & $6(31.6)$ & $6(31.6)$ & $12(31.6)$ \\
\hline Others $^{\mathrm{a}}$ & $5(26.3)$ & $3(15.8)$ & $8(21.1)$ \\
\hline Pain duration & $22.8 \pm 10.9$ & $22.7 \pm 24.2$ & $22.7 \pm 11.8$ \\
\hline NRS average at baseline & $5.3 \pm 1.9$ & $5.3 \pm 2.3$ & $5.3 \pm 2.1$ \\
\hline NRS worst at baseline & $6.6 \pm 1.6$ & $6.7 \pm 2.0$ & $6.7 \pm 1.8$ \\
\hline Strong opioid use & $7(36.8)$ & $8(42.1)$ & $15(39.5)$ \\
\hline HAM-A score & $22.8 \pm 10.9$ & $23.6 \pm 10.3$ & $23.2 \pm 10.4$ \\
\hline
\end{tabular}

Values are presented as mean \pm standard deviation or number (\%).

VR: virtual reality, NRS: numerical rating scale, HAM-A: Hamilton Anxiety Rating.

${ }^{a}$ Other diagnoses included painful peripheral diabetic polyneuropathy ( $\mathrm{n}=3$ in the VR group and $\mathrm{n}=2$ in the control group) and chronic postoperative pain syndrome ( $n=1$ in each group).

Table 2. Procedure-related variables

\begin{tabular}{lccc}
\hline \multicolumn{1}{c}{ Variable } & VR $(\mathrm{n}=19)$ & Control $(\mathrm{n}=19)$ & $P$ value \\
\hline Procedure-related NRS pain score (0-10) & $3.7 \pm 1.4$ & $5.5 \pm 1.7$ & 0.002 \\
Procedure-related anxiety before intervention (1-5) & $3.6 \pm 0.6$ & $3.4 \pm 0.6$ & 0.288 \\
Procedure-related anxiety after intervention (1-5) & $2.5 \pm 0.8$ & $3.2 \pm 0.7$ & 0.010 \\
Difference between pre- and post-procedural anxiety (0-4) & $-1.1 \pm 0.8$ & $-0.2 \pm 0.6$ & $3.6 \pm 1.0$ \\
Satisfaction on pain control for the procedure (1-5) & $3.2 \pm 0.8$ & $4(21.1)$ & 0.001 \\
Patients reporting much and extremely satisfied scored 4 and 5 (\%) & $10(52.6)$ & $13(68.4)$ & 0.091 \\
Patients who requested additive LA during the procedure (\%) & $4(21.1)$ & $6.1 \pm 1.2$ & 0.008 \\
Total procedure time (min) & $31.5 \pm 2.7$ & $30.0 \pm 3.0$ & 0.119 \\
Total stay at the operating room (min) & $20.9 \pm 6.4$ & $25.3 \pm 4.8$ & 0.022 \\
Patients' perception of the stay at the operating room (min) &
\end{tabular}

Values are presented as mean \pm standard deviation or number (\%).

VR: virtual reality, NRS: numerical rating scale, LA: local anesthetics. 
(min) of LSGB and wearing of the VR device were comparable between the groups. However, subjective perception of OR stay (min) was significantly shorter in the VR group (25.3 \pm 4.8 vs. $20.9 \pm 6.4$, respectively; $P=0.022$ ).

Significant correlations existed between the postprocedural NRS and the baseline average NRS pain scores ( $\rho$ $=0.32, P=0.048)$; current strong opioid use ( $\rho=0.32, P=$ $0.048)$; and comorbid anxiety disorder $(\rho=0.49, P=0.002)$ (Table 3). Subjective feedback on the use of VR immersion in the VR group revealed that 12 patients $(63.2 \%)$ wanted to use VR immersion in their next pain procedure, 6 patients (31.6\%) did not make the decision, and 1 patient (5.3\%) declined due to unfamiliarity. The VR application was not interrupted by the physician or the patients in any instance.

\section{Comparison of hemodynamic variables and adverse reactions}

Pre-procedural and post-procedural hemodynamic measurements did not differ between the groups (Table 4). In the VR group, 2 patients (10.5\%) reported transient dizziness at some point during the procedure, but they refused to drop out, maintaining their VR immersion. Another 3 patients ( 1 in the VR and 2 in the control group) complained of discomfort wearing the VR headset in a prone position; however, it was bearable to continue. In the recovery room, 2 patients in the VR group (10.5\%) and 3 patients in the control group (15.8\%) reported transient dizziness along with mild nausea, which disappeared before discharge from the hospital. No other adverse events relating to the VR immersion were observed. No rescue medication was administered to either group during the study.

\section{DISCUSSION}

We conducted an exploratory randomized controlled trial to evaluate the effectiveness of VR immersion combined with conventional skin infiltration compared to skin infiltration alone. To the best of our knowledge, this study was the first one to assess the feasibility of VR in patients in a prone position while performing a painful procedure. The procedure-related pain during LSGB was significantly lower in the VR group $(3.7 \pm 1.4$ in the 11-pointed NRS score) than in the control group (5.5 \pm 1.7 in the 11-point NRS score; $P=0.002$ ), without any severe adverse events associated with the VR immersion during the procedure. Fewer patients required additive LA in the VR group. Although satisfaction with procedural pain control did not differ between the groups, post-procedural anxiety was lower, and patients' subjective perception of their stay in

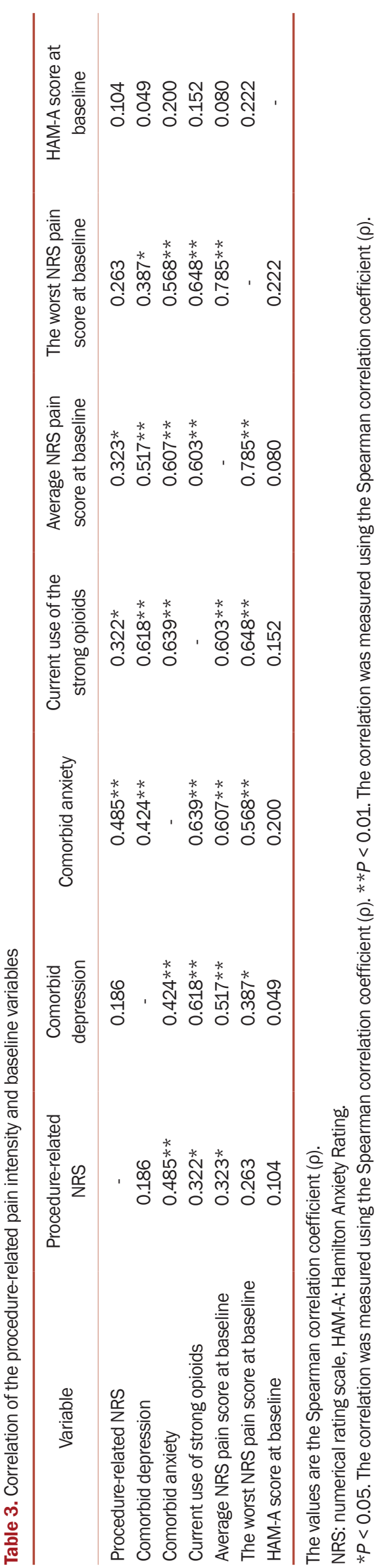

https://doi.org/10.3344/kjp.2021.34.3.304 
Table 4. Vital signs and adverse events

\begin{tabular}{lccr}
\hline \multicolumn{1}{c}{ Variable } & VR $(\mathrm{n}=19)$ & Control $(\mathrm{n}=19)$ & $P$ value \\
\hline Pre-systolic pressure $(\mathrm{mmHg})$ & $143.6 \pm 26.8$ & $142.7 \pm 27.1$ & 0.914 \\
Post-systolic pressure $(\mathrm{mmHg})$ & $139.1 \pm 24.9$ & $139.2 \pm 27.5$ & 0.990 \\
Pre-diastolic pressure $(\mathrm{mmHg})$ & $79.4 \pm 13.2$ & $73.1 \pm 15.9$ & 0.194 \\
Post-diastolic pressure $(\mathrm{mmHg})$ & $78.5 \pm 13.0$ & $70.2 \pm 15.3$ & 0.079 \\
Pre-mean pressure $(\mathrm{mmHg})$ & $100.8 \pm 15.3$ & $96.3 \pm 17.9$ & 0.411 \\
Post-mean pressure $(\mathrm{mmHg})$ & $98.7 \pm 13.9$ & $93.2 \pm 16.1$ & 0.267 \\
Pre-heart rate (beats/min) & $76.4 \pm 14.7$ & $70.2 \pm 13.9$ & 0.197 \\
Post-heart rate (beats/min) & $72.7 \pm 22.1$ & $70.9 \pm 13.4$ & 0.775 \\
Pre $\mathrm{O}_{2}$ saturation (\%) & $98.2 \pm 1.8$ & 0.595 \\
Post- $\mathrm{O}_{2}$ saturation $(\%)$ & $98.1 \pm 2.3$ & $97.8 \pm 2.4$ & 0.577 \\
Adverse events & $3(15.8)$ & $97.6 \pm 2.3$ & $>0.999$ \\
\hline
\end{tabular}

Values are presented as mean \pm standard deviation or number (\%).

VR: virtual reality.

the OR was shorter in the VR group than in the control group. Almost two-thirds of patients (63.2\%) wanted to undergo VR immersion again in their following pain procedure.

Despite their minimal invasiveness, pain interventions, including LSGB, are still painful and distressing for patients with chronic pain [5]. Pain physicians have attempted to alleviate procedure-related pain and anxiety during their interventions. VR is one of the emerging options for rapid implementation in various clinical settings, ranging from managing acute procedure-related pain to rehabilitating chronic pain conditions $[8,9]$. It has been used in needle-related procedures, anesthesia administration, and other painful interventions, including dressing changes for burn wounds [10-16]. In recent studies, VR was suggested to positively modulate chronic intractable pain [21], and was self-administered at home to manage chronic low back pain during COVID-19 [22]. However, few studies have reported the use of VR in reducing acute procedure-related pain of the elderly in outpatient pain practice. Presumably, there have been several limitations in adopting VR in outpatient pain settings. First, most fluoroscopy-guided procedures in pain practice require patients to be in the prone position, which may hinder the comfortable use of the current VR device. Second, pain physicians may want to ensure instant patient response during the procedure. Accordingly, VR immersion may impede the interaction between patients and their physicians. Besides, many studies investigated the use of VR for procedural pain targets in pediatric cases, adolescents, or young adults rather than the elderly [9,23]; however, most of the subjects in pain practice are elderly, as in our study, which may discourage the clinical application of VR in routine practice. Recently, Brown et al. [24] reported lower anxiety scores in patients with VR immersion than the controls at their outpatient spine center visits. However, in their study, patients experienced VR in a sitting position, but not in a prone position, ahead of the spine procedures [24]. Therefore, our study firstly showed the benefit of VR in procedure-related pain control for elderly patients undergoing minimally-invasive pain procedures in a prone position in outpatient pain practice. Although three patients in our study felt discomfort wearing the VR headset in a prone position, it was bearable. We suggest that lighter and more user-friendly VR hardware can extend the use of VR technology in various medical environments.

The types of VR for reducing procedural pain differ according to the subject's environment, ranging from nonimmersive to fully-immersive approaches [9]. In previous studies, fully immersive VR distraction games were often used to relieve acute procedural pain, resulting in mostly successful outcomes [9]. Such VR distraction games might draw the patients' attention away from adverse stimuli by exposing them to rich sensory stimuli, creating a realistic experience $[25,26]$. Instead of VR distraction games, an immersive VR program commercially developed for medical hypnosis was used in our study, resulting in reduced procedural pain and anxiety. VR hypnosis has rarely been studied in procedure-related pain control compared to VR distraction games [27]. Previously, Konstantatos et al. [28] used an 18-minute VR self-hypnosis program in burn patients undergoing awake dressings changes. However, their results showed increased pain after dressing in the VR hypnosis group, which suggested that a single session of VR hypnosis for burn patients undergoing an 18-minute dressing was not enough to reduce procedural pain. Although we used a VR hypnosis program, the pain-evoking procedure of LSGB lasted only about 6 minutes (shown in Table 2). Although our patients might not have been fully immersed in VR hypnosis during the first 6 minutes, they could experience it for the remaining 20 minutes without painful external stimuli, which probably contributed to 
differences between our study and Konstantatos's. Because there are numerous pain procedures with different durations and levels of invasiveness, further studies are necessary to identify the types of VR, which effectively attenuate procedural pain and anxiety in patients.

Although VR immersion reduced procedural pain with a statistical significance, the difference in the 11-point NRS pain scores between the VR and control groups was only 1.6 points, which did not meet our preset level that was a reduction of $2 / 10$ or more in the 11-point NRS pain score. Nonetheless, the IMMPACT (Initiative on Methods, Measurement, and Pain Assessment in Clinical Trials) guideline stated that a smaller difference, of less than $2 / 10$ between groups, might indicate a significant difference [19]. Besides the procedural pain reduction, VR immersion alleviated post-procedural anxiety in our study, similar to other VR studies $[8,9]$. In contrast to the former VR study [16], satisfaction with procedural pain control was not different between the VR and the control groups in the present study. We assume that this may be related to the interactive use of additive LA in conscious patients in both groups, responding to patients' requirements during the procedure. VR technology is affordable, safer, and more user-friendly than IV sedation in busy outpatient pain settings. Therefore, there is no reason not to embrace this modality in pain practice.

Beyond the acute procedural pain control, VR technology is available for chronic pain management, such as phantom limb pain, complex regional pain syndrome, and fibromyalgia, suggesting its potential neuromodulatory effect $[8,27,29,30]$. Although it was not shown in our results, when we compared the NRS pain scores in patients' follow-up visits, patients' average and worst pain intensity were not different between the groups at one month. We presume that a single session of VR intervention was not adequate to deduce the intensity of chronic pain, in contrast to previous studies that demonstrated the impact of multiple VR sessions [29-31]. The use of VR in conjunction with conventional chronic pain management warrants further investigation, incorporating different types of VR, interactivity, embodiment, and duration or frequencies of VR intervention according to the target goals of use in pain medicine.

The current study has several limitations. First, our study was a single-center trial with a small sample size, which might limit its external validity. The effect of VR intervention may differ in various clinical settings. Additional studies are needed to spread VR technology in other minimally invasive pain procedures, such as relatively simple (e.g., single epidural injection) or complex procedures (e.g., multi-level heat radiofrequency neurodestruction). Second, although we used sham intervention in the control group wearing the VR device, patients and physicians could not be blinded. Add-on design (VR + LA injection vs. LA injection standalone) without blinding entails a placebo effect. To investigate that effect, it might be better to include a 3rd group not wearing the VR device, undergoing the procedure in the usual way in pain practice. Third, we measured patients' anxiety using the HAM-A score at baseline and a single 5-pointed Likert scale before and after the procedures. The Beck Anxiety Inventory may facilitate the evaluation of changes in procedure-related anxiety in detail [32]. Fourth, most of our patients were naïve to the VR application. The results could potentially differ if our participants were familiar with VR technology or the particular VR program. Finally, regarding the VR applications, we used the commercial VR hypnosis program rather than VR distraction games. Hypnosis would require some time of induction before the onset of the effect; however, we did not consider it in this study. Further, it is necessary to explore whether different VR programs work differently and investigate the types of VR that are the most effective to control procedural pain in the elderly during minimally invasive pain interventions.

In conclusion, the procedure-related pain during LSGB, and anxiety after the procedure, were significantly lower in patients exposed to VR, along with skin infiltration, than in those with skin infiltration alone in outpatient pain practice. No severe adverse events were associated with the VR immersion during the procedure. Although patients' degree of satisfaction with their procedural pain control was similar, the additive requirements of LA during the procedure were fewer in patients exposed to VR immersion. This study suggests that VR immersion can be safely used as a novel adjunct to conventional skin block for managing procedural pain and anxiety in elderly patients undergoing fluoroscopy-guided spine procedures in a prone position.

\section{ACKNOWLEDGMENTS}

We wish to thank Diane Jooris, co-founder and CEO of Oncomfort SA (Wavre, Belgium), who provided the Virtual Reality hypnosis program free of charge. She and the company had no role in study design, data collection and analysis, decision to publish, or preparation of the manuscript.

\section{CONFLICT OF INTEREST}

No potential conflict of interest relevant to this article was reported. 


\section{FUNDING}

No funding to declare.

\section{ORCID}

Young Joo, https://orcid.org/0000-0001-5104-9486

Eun-Kyung Kim, https://orcid.org/0000-0002-8938-0366

Hyun-Gul Song, https://orcid.org/0000-0002-4867-0818

Haesun Jung, https://orcid.org/0000-0002-4928-6930

Hanssl Park, https://orcid.org/0000-0002-8936-0373

Jee Youn Moon, https://orcid.org/0000-0001-5551-7750

\section{REFERENCES}

1. Castañeda AM, Lee CS, Kim YC, Lee D, Moon JY. Addressing opioid-related chemical coping in long-term opioid therapy for chronic noncancer pain: a multicenter, observational, cross-sectional study. J Clin Med 2018; 7: 354.

2. Semeru GM, Halim MS. Acceptance versus catastrophizing in predicting quality of life in patients with chronic low back pain. Korean J Pain 2019; 32: 22-9.

3. Foster NE, Anema JR, Cherkin D, Chou R, Cohen SP, Gross DP, et al.; Lancet Low Back Pain Series Working Group. Prevention and treatment of low back pain: evidence, challenges, and promising directions. Lancet 2018; 391: 2368-83.

4. Kim EJ, Moon JY, Park KS, Yoo da H, Kim YC, Sim WS, et al. Epidural steroid injection in Korean pain physicians: a national survey. Korean J Pain 2014; 27: 35-42.

5. Ryu JH, Lee CS, Kim YC, Lee SC, Shankar H, Moon JY. Ultrasound-assisted versus fluoroscopic-guided lumbar sympathetic ganglion block: a prospective and randomized study. Anesth Analg 2018; 126: 1362-8.

6. Angelini G, Ketzler JT, Coursin DB. Use of propofol and other nonbenzodiazepine sedatives in the intensive care unit. Crit Care Clin 2001; 17: 863-80.

7. Shafer A. Complications of sedation with midazolam in the intensive care unit and a comparison with other sedative regimens. Crit Care Med 1998; 26: 947-56.

8. Chuan A, Zhou JJ, Hou RM, Stevens CJ, Bogdanovych A. Virtual reality for acute and chronic pain management in adult patients: a narrative review. Anaesthesia 2021; 76: 695-704.

9. Won AS, Bailey J, Bailenson J, Tataru C, Yoon IA, Golianu B. Immersive virtual reality for pediatric pain. Children (Basel) 2017; 4: 52.

10. Hoffman HG, Doctor JN, Patterson DR, Carrougher GJ, Furness TA 3rd. Virtual reality as an adjunctive pain control during burn wound care in adolescent patients. Pain 2000; 85: 305-9.

11. Brown NJ, Kimble RM, Rodger S, Ware RS, Cuttle L. Play and heal: randomized controlled trial of Ditto ${ }^{\mathrm{mm}}$ intervention efficacy on improving re-epithelialization in pediatric burns. Burns 2014; 40: 204-13.

12. Gold JI, Kim SH, Kant AJ, Joseph MH, Rizzo AS. Effectiveness of virtual reality for pediatric pain distraction during i.v. placement. Cyberpsychol Behav 2006; 9: 207-12.

13. Caruso TJ, George A, Menendez M, De Souza E, Khoury M, Kist MN, et al. Virtual reality during pediatric vascular access: a pragmatic, prospective randomized, controlled trial. Paediatr Anaesth 2020; 30: 116-23.

14. Yamashita Y, Shimohira D, Aijima R, Mori K, Danjo A. Clinical effect of virtual reality to relieve anxiety during impacted mandibular third molar extraction under local anesthesia. J Oral Maxillofac Surg 2020; 78: 545.el-6.

15. Chan PY, Scharf S. Virtual reality as an adjunctive nonpharmacological sedative during orthopedic surgery under regional anesthesia: a pilot and feasibility study. Anesth Analg 2017; 125: 1200-2.

16. Moon JY, Shin J, Chung J, Ji SH, Ro S, Kim WH. Virtual reality distraction during endoscopic urologic surgery under spinal anesthesia: a randomized controlled trial. J Clin Med 2018; 8: 2.

17. BinDhim NF, Shaman AM, Alhawassi TM. Confirming the one-item question Likert Scale to measure anxiety. Internet J Epidemiol [Internet]. 2013 [cited 2018 Sep]; 11: 1-4. Available from: https://print.ispub.com/api/0/ispub-article/14525.

18. Matza LS, Morlock R, Sexton C, Malley K, Feltner D. Identifying HAM-A cutoffs for mild, moderate, and severe generalized anxiety disorder. Int J Methods Psychiatr Res 2010; 19: 223-32.

19. Dworkin RH, Turk DC, Farrar JT, Haythornthwaite JA, Jensen MP, Katz NP, et al.; IMMPACT. Core outcome measures for chronic pain clinical trials: IMMPACT recommendations. Pain 2005; 113: 9-19.

20. Furman E, Jasinevicius TR, Bissada NF, Victoroff KZ, Skillicorn R, Buchner M. Virtual reality distraction for pain control during periodontal scaling and root planing procedures. J Am Dent Assoc 2009; 140: 1508-16.

21. SolcàM, Krishna V, Young N, Deogaonkar M, Herbelin B, Orepic $\mathrm{P}$, et al. Enhancing analgesic spinal cord stimulation for chronic pain with personalized immersive virtual reality. Pain 2021; 162: 1641-9.

22. Darnall BD, Krishnamurthy P, Tsuei J, Minor JD. Self-administered skills-based virtual reality intervention for chronic pain: randomized controlled pilot study. JMIR Form Res 2020; 4: el7293.

23. Sharar SR, Carrougher GJ, Nakamura D, Hoffman HG, Blough DK, Patterson DR. Factors influencing the efficacy of virtual reality distraction analgesia during postburn physical therapy: preliminary results from 3 ongoing studies. Arch Phys Med Rehabil 2007; 88(12 Suppl 2): S43-9.

24. Brown L, Chen ET, Binder DS. The use of virtual reality for 
Peri-procedural pain and anxiety at an outpatient spine clinic injection visit: an exploratory controlled randomized trial. Am J Transl Res 2020; 12: 5818-26.

25. Walker MR, Kallingal GJ, Musser JE, Folen R, Stetz MC, Clark JY. Treatment efficacy of virtual reality distraction in the reduction of pain and anxiety during cystoscopy. Mil Med 2014; 179: 891-6.

26. Frey DP, Bauer ME, Bell CL, Low LK, Hassett AL, Cassidy RB, et al. Virtual reality analgesia in labor: the VRAIL pilot studya preliminary randomized controlled trial suggesting benefit of immersive virtual reality analgesia in unmedicated laboring women. Anesth Analg 2019; 128: e93-6.

27. Rousseaux F, Bicego A, Ledoux D, Massion P, Nyssen AS, Faymonville ME, et al. Hypnosis associated with 3D immersive virtual reality technology in the management of pain: a review of the literature. J Pain Res 2020; 13: 1129-38.

28. Konstantatos AH, Angliss M, Costello V, Cleland H, Stafrace $\mathrm{S}$. Predicting the effectiveness of virtual reality relaxation on pain and anxiety when added to PCA morphine in patients having burns dressings changes. Burns 2009; 35: 491-9.

29. SolcàM, Ronchi R, Bello-Ruiz J, Schmidlin T, Herbelin B, Luthi $\mathrm{F}$, et al. Heartbeat-enhanced immersive virtual reality to treat complex regional pain syndrome. Neurology 2018; 91: e479-89.

30. Garcia-Palacios A, Herrero R, Vizcaíno Y, Belmonte MA, Castilla D, Molinari G, et al. Integrating virtual reality with activity management for the treatment of fibromyalgia: acceptability and preliminary efficacy. Clin J Pain 2015; 31: 564-72.

31. Matheve T, Bogaerts K, Timmermans A. Virtual reality distraction induces hypoalgesia in patients with chronic low back pain: a randomized controlled trial. J Neuroeng Rehabil 2020; 17: 55.

32. Beck AT, Steer RA. Relationship between the Beck anxiety inventory and the Hamilton anxiety rating scale with anxious outpatients. J Anxiety Disord 1991; 5: 213-23. 